\title{
Limb Reduction Defects after Prenatal Inhibition of Nitric Oxide Synthase in Rats
}

\author{
ROBERT L. PIERCE, MARIA R. PIERCE, HAIYAN LIU, PHILIP J. KADOWITZ, AND \\ MARK J. S. MILLER \\ Department of Pediatrics and Pharmacology [R.L.P., M.R.P., H.L., P.J.K.], Tulane University Medical \\ Center, New Orleans, Louisiana 70112 and Department of Pediatrics [M.J.S.M.], Louisiana State \\ University Medical Center, New Orleans, Louisiana 70112
}

\begin{abstract}
To determine the influence of nitric oxide (NO) on vascular tone during fetal development, timed pregnant rats received the NO synthase inhibitor $N^{\mathrm{G}}$-nitro-L-arginine methyl ester for consecutive 4,7 , or $14 \mathrm{~d}$ before parturition (postorganogenesis). Offspring demonstrated limb reduction defects (incidence, 53\%) involving either or both hindlimbs, whereas forelimbs were uniformly spared. Defects were dose-dependent but independent of the duration of administration occurring with equal frequency in 4-, 7-, and 14-d treatment groups. Histologic analysis revealed features characteristic of vascular disruption with hemorrhagic necrosis and loss of structure. The defects were prevented by concurrent maternal administration of L-arginine or the NO donors $S$-nitroso- $N$-acetyl-penicillamine and sodium nitroprusside. Defects were not seen after prenatal treatment with aminoguanidine. To study basal and agonist-mediated NO release, newborn femoral and brachial arteries were cannulated with a glass micropipette under constant pressure, and changes in intraluminal diameter (micrometers) were measured in response to acetylcholine and the NO synthase inhibitor $N_{\omega}$-nitro-L-arginine.
\end{abstract}

\section{ABSTRACT}

Newborn femoral and brachial vessels demonstrated a dramatic (59\%) decrease in resting diameter compared with adult vessels (16\%). These findings suggest that basal NO release is upregulated during fetal development concurrent with the processes that increase maternal NO release. The data also suggest that up-regulation of NO release occurs throughout the fetal systemic circulation and is not restricted to hindlimbs. This is the first study to demonstrate inhibition of NO release in the pathogenesis of limb reduction defects. (Pediatr Res 38: 905-911, 1995)

NO, nitric oxide

\section{Abbreviations}

NOS, nitric oxide synthase

LRD, limb reduction defects

L-NAME, $N^{\mathrm{G}}$-nitro-L-arginine methyl ester

L-NNA, $N_{\omega}$-nitro-L-arginine

SNP, sodium nitroprusside

SNAP, $S$-nitroso-acetylpenicillamine
$\mathrm{NO}$ is a potent vasodilator produced by vascular endothelium and smooth muscle from the amino acid L-arginine by NOS. Several isoforms of NOS have been isolated from brain (type I), macrophages (type II), and vascular endothelium (type III). Both the brain and endothelial isoforms are calcium/ calmodulin-dependent enzymes which are expressed under physiologic conditions (constitutive). The inducible isoform, in contrast, is calcium/calmodulin-independent and is expressed in vascular smooth muscle solely in response to various cytokines or endotoxins. The physiologic regulation of vascular tone by the constitutive isoform generally occurs through two distinct pathways: continuous or basal NO release and that which is generated in response to various agonists or physical

Received September 27, 1994; accepted July 6, 1995

Robert L. Pierce, M.D., Department of Pediatrics, Section of Cardiology, Tulane Medical Center, 1430 Tulane Ave., New Orleans, LA 70112.

Supported by the Louisiana Chapter of the American Heart Association (M.R.P.), a National Institutes of Health Basic Research Science Grant (R.L.P.), and National Institutes of Health Grant RO1 HD31885 (M.J.S.M.). stimuli such as shear stress $(1,2)$. A number of L-arginine analogs such as L-NAME and L-NNA have been identified which effectively inhibit both the constitutive and inducible isoforms. Aminoguanidine predominately inhibits the inducible NOS isoform.

The mechanisms that regulate the changes in vascular tone that characterize the dynamic nature of the perinatal circulation are incompletely understood. Even less is known about factors governing vascular tone during normal fetal growth and development. Common complications of premature birth such as intraventricular hemorrhage, persistent fetal circulation, and necrotizing enterocolitis are believed to be related to pathology of the fetal vasculature. To determine the role of endothelial NO release during normal fetal growth and development, we recently investigated the effects of chronic early third trimester inhibition of NO release with the L-arginine analog, L-NAME, in timed pregnant Sprague-Dawley rats. Although L-NAME produced significant intrauterine growth retardation (3), the most remarkable findings were LRD involving the hindlimbs. 
The hindlimbs demonstrated features characteristic of vascular disruption with evidence of grossly hemorrhagic tissue and loss of structure. Furthermore, although either or both hindlimbs were involved, the forelimbs were uniformly spared. Although LRD have been produced by several teratogens (4), the effect is almost always seen early in the first trimester period of organogenesis when the fetus is most vulnerable to birth defects (5). The production of LRD by L-NAME during the third trimester was an unusual teratogenic effect with several intriguing implications regarding the role of endothelial NO release in the systemic circulation during late fetal development.

Increased maternal and placental NO release during pregnancy has been previously demonstrated $(6,7)$. Our preliminary findings suggested that NO release may also be increased during late fetal development, specifically in the hindlimbs. The preliminary data required further investigation of the teratogenic effects of L-NAME and further determination of a process specific for inhibition of fetal endothelial NO release. L-NAME is a nonspecific inhibitor of both inducible and constitutive isoforms. In addition, L-arginine is a semiessential amino acid, and disruptions might develop due to inhibition of L-arginine uptake during the relative period of rapid growth characteristic of the third trimester. Both in vivo and in vitro studies were undertaken with the following specific goals: 1 ) to determine the effect of both the dose and duration of L-NAME administration on the incidence LRD;2) to determine whether the LRD were specific for inhibition of constitutive NOS and endothelial NO release as suggested by the histologic finding of hemorrhagic necrosis; and 3) to determine whether basal or acetylcholine-mediated NO release is increased in isolated hindlimb vessels from newborns compared with adult animals which might explain vulnerability to vascular disruptions.

\section{METHODS}

All animal treatments and procedures were preapproved by the Louisiana State University Medical School Committee on Research Animal Welfare. They were maintained in the Animal Care Facilities of the Louisiana State University Medical School under veterinary supervision on standard rat pellet diet ad libitum and kept on a 12-h light/dark cycle. Rats were chosen as the study model because they manifest hemodynamic alterations during gestation closely resembling those of a gravid human (8). All chemicals were obtained from Sigma Chemical Co. (St. Louis, MO). Acetylcholine and SNP were prepared fresh daily in normal saline stock solutions. U46619 and indomethacin stock solutions were prepared in 95\% ethanol. Ethanol vehicle from stock solutions did not affect vascular tone. L-NNA was dissolved in acidified normal saline with a final $\mathrm{pH}$ adjusted to 7.0 .

\section{In Vivo Studies}

Timed pregnant rats (Holtzman, Harlan Sprague-Dawley) were obtained on gestational d 6 of a $22-d$ gestation. Four to six dams were randomly assigned to the treatment groups described below (10-16 pups per litter). The in vivo studies were conducted in four parts. Part 1 determines whether the inci- dence of LRD increases with dose and duration of prenatal L-NAME treatment (i.e. second through third trimester (14 prenatal days) versus late third trimester (4 prenatal days). Part 2 determines whether LRD occur with postnatal L-NAME treatment. Part 3 determines whether concurrent treatment with the NO precursor (L-arginine) or NO donors SNP or SNAP alters the incidence of LRD produced by L-NAME. Part 4 determines whether selective inhibition of inducible NOS with aminoguanidine results in LRD.

Part 1: Dose and duration of NOS inhibition with L-NAME and $\mathbf{L R D}$. The dose range of L-NAME used in this study was based on our previous experience with doses of L-NAME which reduce the elevated NO production associated with experimental inflammatory bowel disease (9), as well as a number of reports evaluating hypertension induced by chronic reductions of NO synthesis $(10,11)$. The oral route of administration of the NOS inhibitors has been shown to be a simple and reliable approach to the chronic reduction of NOS in vivo (9-11). Daily fluid consumption was followed in two to three animals per cage and an estimated daily dose of L-NAME between 54 to $180 \mathrm{mg} / \mathrm{kg} / \mathrm{d}$ was obtained with concentrations of 0.3 and $1.0 \mathrm{mg} / \mathrm{mL}$ L-NAME in the drinking water, respectively. Dams were studied at 6 through 19 and 16 through 19 $\mathrm{d}$ of gestation, corresponding to 14 and $4 \mathrm{~d}$ of treatment, respectively. For each gestational period studied, dams were randomly assigned one of three treatment groups $(0.3$ and 1.0 $\mathrm{mg} / \mathrm{mL}$ L-NAME or water only controls). On gestational d 20 , dams were given an overdose of intraperitoneal sodium pentobarbital. Fetuses were removed from the uterus and inspected for LRD. LRD were defined by partial or complete reduction in the size of a limb and by the presence of structural or morphologic abnormalities. Control and altered limbs were fixed in $10 \%$ paraformaldehyde, paraffin-embedded, and stained with hematoxylin and eosin for histologic evaluation.

Part 2: Postnatal L-NAME. Because the quantity of $\mathrm{L}-$ NAME excreted in breast milk is not known, two different routes of administering L-NAME were studied in newborn rats. In the first, L-NAME was administered for 14 postnatal days indirectly in maternal breast milk $(1.0 \mathrm{mg} / \mathrm{mL}$ of maternal drinking water). In a second group, L-NAME was administered through daily intraperitoneal injection $(180 \mathrm{mg} / \mathrm{kg})$ in $0.1 \mathrm{~mL}$ of saline. A third group served as water only controls in the breast-fed group, and a fourth group received intraperitoneal injections of $0.1 \mathrm{~mL}$ saline. Newborns were examined daily for LRD.

Part 3: L-NAME plus L-arginine, SNP, or SNAP. Timed pregnant rats were randomly assigned to one of four treatment groups (timing and doses based on results of part 1). The first received L-NAME at $1.0 \mathrm{mg} / \mathrm{mL}$ plus the $\mathrm{NO}$ precursor $\mathrm{L}-$ arginine at $0.03 \mathrm{~g} / \mathrm{mL}$, which approximates $180 \mathrm{mg} / \mathrm{kg} / \mathrm{d}$ and $5.4 \mathrm{~g} / \mathrm{kg} / \mathrm{d}$, respectively, in the drinking water. The second group received $\mathrm{L}-\mathrm{NAME}$ at $1.0 \mathrm{mg} / \mathrm{mL}$ in the drinking water plus the NO donor SNP, by s.c. implantation of osmotic minipumps (Alza Corp., Palo Alto, CA) at 1.0, 3.0, and 10 $\mu \mathrm{g} / \mathrm{kg} / \mathrm{min}$. The third group received $\mathrm{L}-\mathrm{NAME}$ at $1.0 \mathrm{mg} / \mathrm{mL}$ in the drinking water plus the NO donor SNAP, by s.c. implantation of osmotic minipumps at $1.0,3.0$, and $10 \mu \mathrm{g} / \mathrm{kg} / \mathrm{min}$. The fourth group was a water-only control. After $7 \mathrm{~d}$ of 
prenatal treatment (gestational d 13-19), on gestational d 20 dams received an overdose of intraperitoneal sodium pentobarbital. Fetuses were removed and inspected for LRD.

Part 4: Selective inhibition of inducible NOS with aminoguanidine. Pregnant dams were randomly assigned to one of two treatment groups. The first received the selective inducible NOS inhibitor, aminoguanidine, at $1.0 \mathrm{mg} / \mathrm{mL}$ for 7 prenatal days (gestational d 13-19), which approximates $180 \mathrm{mg} / \mathrm{kg} / \mathrm{d}$. The second group was a water-only control. On gestational d 20, dams were given an overdose of intraperitoneal sodium pentobarbital. Fetuses were removed and inspected for LRD.

\section{In Vitro Studies}

Adult and newborn rats (3-7 d of age) were killed after intraperitoneal sodium pentobarbital, and either the femoral or brachial artery was rapidly removed and placed in Krebsbicarbonate buffer at $3-5^{\circ} \mathrm{C}$ equilibrated with a $95 \% \mathrm{O}_{2} / 5 \%$ $\mathrm{CO}_{2}$ gas mixture. Vessels were carefully cleaned of surrounding fat and adventitia and transferred to the myograph chamber. Vessels were cannulated with a glass micropipette of known internal diameter and then gently flushed to remove remaining blood before the distal end was tied with surgical nylon monofilament to create a blind pouch configuration. Vessels were then allowed to equilibrate for $45 \mathrm{~min}$ as temperature was slowly raised to $37^{\circ} \mathrm{C}$ and intraluminal pressure was slowly raised to $30 \mathrm{~mm} \mathrm{Hg}$ for newborn vessels and 100 $\mathrm{mm} \mathrm{Hg}$ for adult vessels. These pressures were previously determined to provide maximal response to $\mathrm{KCl}$ contracture. Cannulated vessels were illuminated on an inverted microscope and displayed through a camera connected to a dimension analyzer (Living Systems Inc., Burlington, VT) that provides a constant digital display of lumen diameter and wall thickness. A microliter peristaltic pump with an automatic pressure servocontrol maintained constant intraluminal pressure throughout the study $( \pm 1 \mathrm{~mm} \mathrm{Hg})$. After equilibration, vessels were required to maintain a stable intraluminal pressure (manual setting of pressure servocontrol) as assurance that no flow or leaks were present. Both pressure and lumen diameter were continuously recorded on a strip chart recorder. All chemicals were added to the buffer superfusion reservoir and allowed to recirculate through the myograph chamber (total volume, $80 \mathrm{~mL}$ ). The myograph chamber was continuously perfused at $37^{\circ} \mathrm{C}$ with Kreb's bicarbonate buffer equilibrated with $21 \% \mathrm{O}_{2}, 5 \% \mathrm{CO}_{2}$, and balance with $\mathrm{N}_{2}$ at $\mathrm{pH} 7.4$ which contained (in mM) $110 \mathrm{NaCl}, 5 \mathrm{KCl}, 2.5 \mathrm{CaCl}, 1 \mathrm{MgSO}_{4}, 1$ $\mathrm{KHPO}_{4}, 24 \mathrm{NaHCO}_{3}, 10$ dextrose, 0.02 EDTA, and 0.01 indomethacin. Both superfusate flow rate and gas were adjusted to maintain $\mathrm{pH}$ and temperature which were continuously monitored through a YSI temperature probe directly in the myograph and a $\mathrm{pH}$ probe placed in the reservoir. Vessels studied in both newborn and adult animals demonstrated passive dilation to increments in intraluminal pressure with no significant myogenic response.

As a measure of agonist-independent maximal contractile response all vessels were initially challenged with $60 \mathrm{mM} \mathrm{KCl}$. Vessels that demonstrated less than 50\% decrease in resting diameter were discarded. Higher doses of $\mathrm{KCl}$ completely obliterated intraluminal diameter in all vessels. After washout of $\mathrm{KCl}$, all vessels were submaximally preconstricted with 30 $\mathrm{nM}$ of the thromboxane mimic U46619. U46619 also served as a basis for comparison of agonist-mediated responses with the change in resting diameter produced by L-NNA. To analyze basal NO release after washout of U46619, vessels were challenged with incremental doses of L-NNA. Acetylcholine served to determine endothelial, receptor-mediated NO release, respectively. Inasmuch as acetylcholine is not a pure NO agonist but activates additional mechanisms, NO-specific responses were quantified by subsequent inhibition of peak acetylcholine responses with L-NNA. SNP responses were determined as a measure of endothelium-independent, guanylate cyclase-mediated tone.

Statistical analysis. Incidence of disruptions in the treated versus untreated rat pups were compared by $\chi^{2}$ testing (12). For in vitro studies, vessel responses were compared with a two-way analysis of variance where a $p<0.05$ value was considered to represent a statistically significant difference between the compared values. Only one vessel from each animal was studied.

\section{RESULTS}

In vivo studies. Maternal health did not appear to be compromised by the NOS inhibitors. Maternal weight gain was comparable in each group, averaging $14.8 \pm 1.2 \mathrm{~g} / \mathrm{d}$. Fluid consumption was also comparable in each group, averaging $184 \pm 27 \mathrm{~mL} / \mathrm{kg} / \mathrm{d}$. No LRD were observed in fetuses of control dams ( $>500$ control fetuses). The incidence of LRD in fetuses prenatally treated with the selective inhibitor of the inducible NOS isoform, aminoguanidine, was also zero (out of 84 fetuses). In all aspects, aminoguanidine-treated animals could not be distinguished from control animals. L-NAMEinduced LRD were influenced by the dose administered $(0.3$ versus $1.0 \mathrm{mg} / \mathrm{mL})(p>0.05)$ but not by the duration of prenatal exposure, occurring with equal frequency after 4,7 , or $14 \mathrm{~d}$ of prenatal treatment (Fig. 1). The disruptions were found only in the hindlimbs, and it was observed in either leg or both, varying in severity from disruptions of toes to complete absence of proximal thigh (Fig. $2 A$ ). Histologically the L-NAMEtreated limbs revealed a hemorrhagic necrosis, with a marked cellular infiltration and loss of structure (Fig. $2 B$ ).

Supplementation with the NO precursor, L-arginine, to dams receiving $1.0 \mathrm{mg} / \mathrm{mL}$ of L-NAME for 7 prenatal days completely inhibited these disruptions ( 0 of 96 fetuses). The addition of the NO donors, SNP and SNAP, to the dams receiving L-NAME significantly reduced the incidence of disruptions in a dose-dependent fashion (Fig. 3, $p<0.05$ ). LRD were not seen during the postnatal period (direct/intraperitoneal: 0 of 62 newborns; indirect/breast milk: 0 of 75). In all parts of the study there were no differences in the number of pups per litter or stillbirths in any treatment group.

In vitro studies. Adult and newborn femoral vessels demonstrated stable resting diameters of $609 \pm 11$ and $177 \pm 9$ $\mu \mathrm{m}$, respectively. Newborn brachial arteries were anatomically somewhat larger than hindlimb vessels with a stable resting diameter of $258 \pm 11 \mu \mathrm{m}$ ( $n=12$ for each vessel group). 


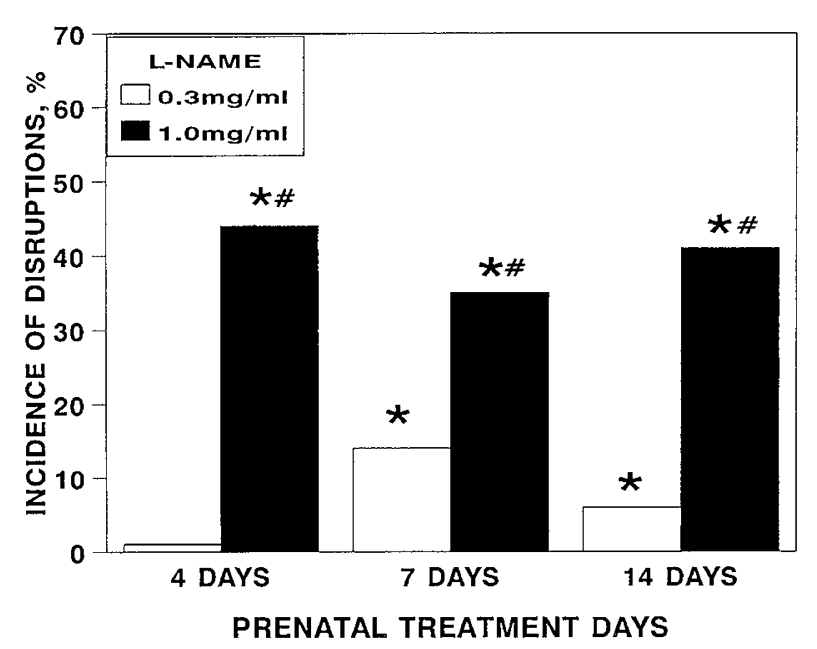

Figure 1. Incidence of hindlimb disruption defects after 4, 7, and $14 \mathrm{~d}$ of prenatal L-NAME at 0.3 and $1.0 \mathrm{mg} / \mathrm{mL}$ drinking water. Values are expressed as mean (percent disruptions) incidence of hindlimb disruptions ( $n=4-6$ litters/group/dose with 10-16 pups per litter). Disruptions occurred in a dosebut not time-dependent fashion, occurring with equal frequency after $4-14 \mathrm{~d}$ of prenatal treatment. The asterisk $(*)$ depicts a significant difference $(p<$ 0.05 ) between prenatally L-NAME-treated $v s$ control fetuses. The number sign (\#) depicts a significant difference $(p<0.05)$ between prenatally L-NAMEtreated fetuses at 0.3 vs $12.0 \mathrm{mg} / \mathrm{mL}$.

Vessels from both age groups demonstrated steady and sustained reductions in resting diameter in response to incremental doses of L-NNA. Most reports on basal NO release have demonstrated little $(<20 \%)$ or no response to L-arginine analogs in resting vessels (2). In adult femoral arteries L-NNA produced a maximal $16 \%$ decrease in resting diameter which is also of similar magnitude to what has been reported in cannulated nonperfused arterioles in rat cremaster muscle (13). Compared with adult vessels, newborn femoral and brachial arteries demonstrated a considerably larger response to L-NNA with a 55 and 59\% decrease in resting diameter, respectively (Fig. 4). Further evidence of the age-related difference in basal NO release is apparent when the L-NNA response is compared with the vasoconstriction produced by $30 \mathrm{nM} \mathrm{U} 46619$ and $60 \mathrm{~mm}$ $\mathrm{KCl}$ (Fig. 5). The response to L-NNA is comparable in magnitude to the response to U46619 in both newborn vessels, whereas in adult femoral arteries the L-NNA response in substantially less in comparison to U46619 ( $p<0.05$ ). Relaxation responses to SNP were quantitatively similar in all three arteries at each concentration (Fig. 6A). Peak relaxation responses to acetylcholine were also quantitatively similar in all three vessels (Fig. 6B). Acetylcholine is known to produce endothelial dependent vasodilatation through NO release and activation of an endothelial hyperpolarization factor (14). To determine that component of the acetylcholine relaxation response specifically mediated by NO release, vessels were subsequently challenged with L-NNA after the peak response to acetylcholine. Both newborn brachial and femoral vessels demonstrated essentially complete $(>90 \%)$ inhibition or reversal of acetylcholine relaxation. Adult femoral vessels in contrast demonstrated only a modest (50\%) inhibition of acetylcholine response (Fig. 7). The L-NNA-mediated inhibition of acetylcholine responses bear striking resemblance to the change in resting diameters produced by L-NNA. In addition, the L-NNA responses did not go below baseline tone which might be expected in response to cumulative inhibition of basal and agonist mediated NO release. As a whole this suggests that the observed responses may primarily reflect differences in basal and not agonist-mediated NO release.

\section{DISCUSSION}

The effects of most teratogens on embryogenesis and fetal development usually occur at a specific time during gestation that defines a vulnerable period for the fetus. This characteristic of a given teratogen, termed stage sensitivity, determines in part the abnormalities that will be observed. Three different stages are commonly recognized. The first extends from the period of fertilization through implantation during which time the embryo is largely composed of undifferentiated omnipotent cells with tremendous reparative capacity. Teratogens acting at this time commonly demonstrate an all on none effect whereby the teratogen is lethal or affects so few cells that the embryo can completely recover. The period of organogenesis is defined by organ formation and differentiation and is the period most sensitive to teratogenic effects and when most anatomic malformations occur. The period after organogenesis is primarily characterized by fetal growth and maturation. Teratogens that affect this period commonly produce cell depletion and growth retardation.

The data presented provide evidence supportive of specific inhibition of endothelial (constitutive NOS) NO release in the pathogenesis of the LRD produced by prenatal administration of L-NAME. The teratogenic effects of L-NAME in this study occurred beyond the period of organogenesis. The toxic effects of L-NAME were not be inhibited or prevented by concurrent administration of $\mathrm{L}$-arginine which is indicative of a process specific for substrate competition. Inhibition of protein synthesis secondary to decreased cellular L-arginine uptake might affect limb growth, but this process would not be expected to be prevented by concurrent administration of NO donors as demonstrated in this study with SNAP and SNP.

Whereas many maturational changes are characterized by functional immaturity with a variable increase with age, the comparatively high NO release in newborns suggests that up-regulation may occur in the fetus concurrent with the mechanisms that govern up-regulation of maternal endothelial NO release. The fact that basal NO release is increased in both femoral and brachial vessels suggests that up-regulation of basal NO release is increased throughout the systemic circulation of the fetus and is not restricted to the hindlimb. LRD were dose-dependent, but were not influenced by the duration of treatment, occurring with equal frequency after 4,7 (previous study), or 14 consecutive days of prenatal treatment (Fig. 1). This result further substantiates a process specific for inhibition of endothelial NO release when one considers the pathophysiology by which L-NAME produces LRD. The histologic finding of hemorrhagic necrosis would suggest that L-NAME produced severe vasoconstriction leading to ischemic tissue death. Hemorrhagic necrosis reflecting cell death from isch- 


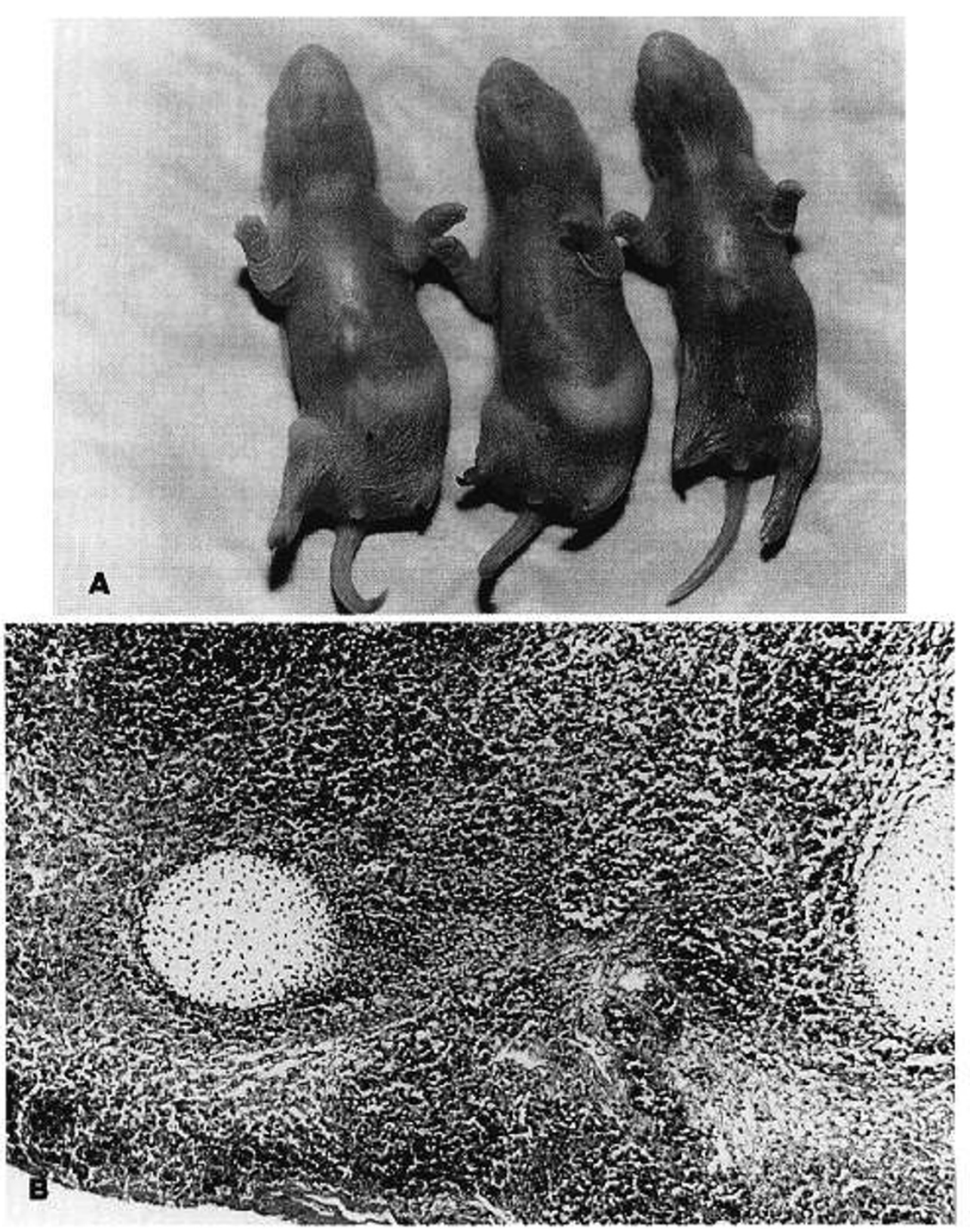

Figure 2. (A) Gross morphology of L-NAME-treated pups (left), showing variable hindlimb involvement with a hemorrhagic appearance of the leg base. (B) Hematoxylin and eosin-stained light microscopy section of the hindlimb with marked hemorrhagic cellular infiltration with thinning of skin and tissue necrosis (original magnification, $\times 200$ ).

emia or infarction would be expected to occur over a relatively short period of time.

One of the primary goals of this study was to investigate mechanisms responsible for localization of vascular disruptions to the hindlimbs of affected offspring. The absence of disruptions after postnatal inhibition of NO release would seem to implicate the intrauterine environment. The most dramatic change that occurs with birth is the transfer of gas exchange from the placenta to the fetus as the lungs are expanded with the first breath. This brings about a substantial increase in oxygen tension and indicates that oxygen substrate availability may play a role in the pathogenesis of vascular disruption in hindlimbs.

The hindlimb circulation of the rat is unique in several respects. Recent studies by Davisson et al. (15) have demon- strated selective autonomic innervation in the hindlimb of the adult rat with very large regional increases in hindlimb blood flow in response to sympathetic activation or stress which can be selectively suppressed with either L-NAME or bretylium. In addition, Gardiner et al. (10) have demonstrated that the initial hypertensive response after i.v. administration of L-NAME is accompanied by marked vasoconstriction in the hindquarter, mesenteric, and renal vascular beds. With time, however, both the mesenteric and renal vasculature return to baseline tone, whereas the hindlimb circulation demonstrates a sustained vasoconstriction. The authors speculated that the hindlimb circulation is unable to compensate for inhibition of NO release. In addition, activation of the baroreceptor reflex in response to L-NAME-mediated hypertension would be ex- 


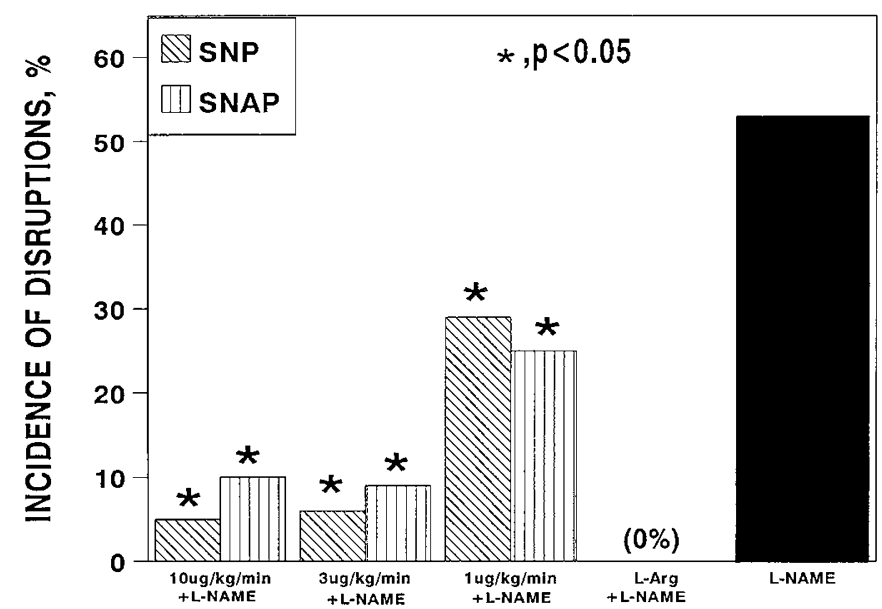

Figure 3. Comparative incidence of hindlimb disruption defects after either 7 $\mathrm{d}$ of prenatal L-NAME $(1.0 \mathrm{mg} / \mathrm{mL}$ drinking waster) alone; L-NAME (1.0 $\mathrm{mg} / \mathrm{mL})$ plus SNP $(1,3$, and $10 \mu \mathrm{g} / \mathrm{kg} / \mathrm{min}$, s.c.) and L-NAME $(1.0 \mathrm{mg} / \mathrm{mL})$ plus SNAP (1, 3 and $10 \mu \mathrm{g} / \mathrm{kg} / \mathrm{min}$, s.c.) $(n=4-8$ litters/group with $10-16$ pups per litter). SNP and SNAP in a dose-dependent fashion reversed the disruptions. The asterisk $(*)$ depicts a significant difference $(p<0.05)$ between prenatally L-NAME-treated alone $v s$ L-NAME plus SNP and SNAP-treated fetuses.

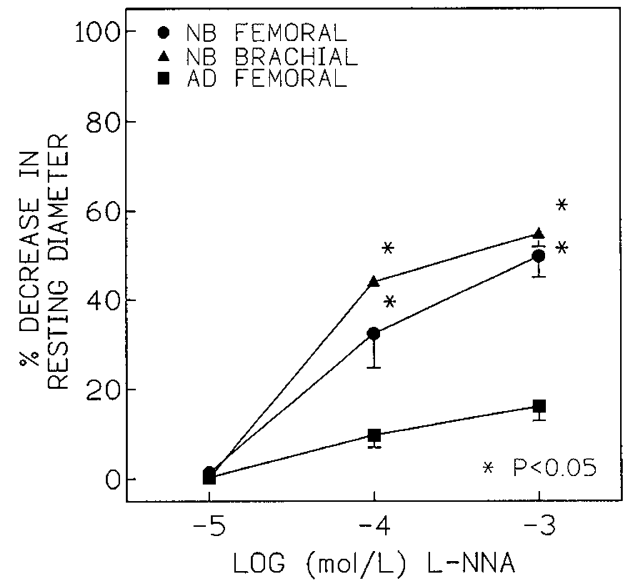

Figure 4. Inhibition of basal NO release determined by percent decrease in resting diameter produced by L-NNA in newborn $(N B)$, femoral $(\bullet)$, brachial $(\mathbf{\Lambda})$, and adult $(A D)$ femoral $(\mathbf{\square})$ arteries cannulated under constant intraluminal pressure. Results are mean $\pm \operatorname{SEM}(n=4$ per vessel group). The asterisk $(*)$ depicts significant difference in newborn femoral and brachial vessels $(p<$ $0.05) v s$ adult femoral artery.

pected to cause a further decrease in hindlimb blood flow in response to inhibition of sympathetic tone.

Systemic hypertension in the fetus after prenatal administration of L-NAME may also play a role in the teratogenic effects observed. Hypertension has been implicated in the pathogenesis of LRD in the edema syndrome in chick embryos seen after exposure to moderated hypoxia (16). Moderate hypoxia in chick embryos produces an osmoregulatory disturbance with hypertension, hypervolemia, and death secondary to vessel rupture and hemorrhage. Leist and Grauwiler (17) have also reported the teratogenic effects of moderate hypoxia in rats produced by uterine artery clamping on gestational $\mathrm{d} 14$. Litter size was dramatically reduced, and offspring demonstrated generalized edema and reduction defects of the snout, forelimbs, and hindlimbs. L-NAME produced no change in liter

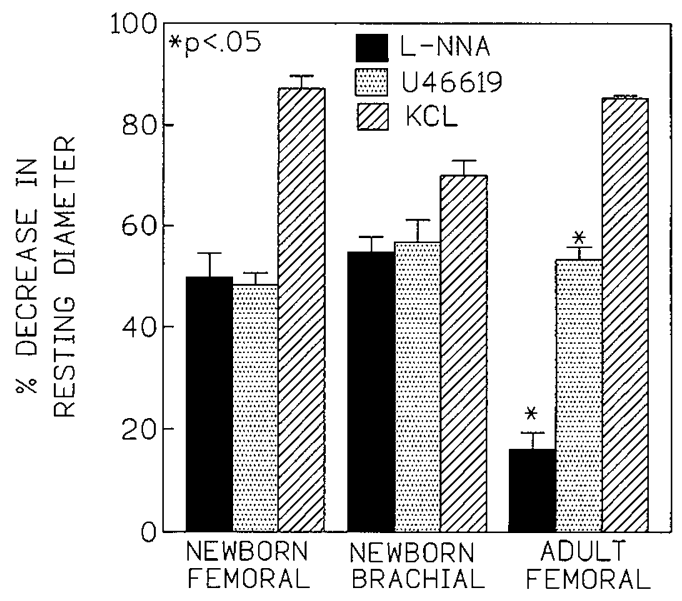

Figure 5. Comparison of age related changes in resting diameter produced by inhibition of basal NO release with L-NNA against responses to agonist dependent (30 $\mu \mathrm{M}$ U46619) and independent, $60 \mathrm{mM}$ KCL responses. The response to U46619 is comparable in the newborn and adult. In contrast, the L-NNA response is equivalent to U46619 in both newborn vessels whereas in the adult the response is notably less $(* p<0.05$ ). Results are mean \pm SEM ( $n$ $=4$ per vessel group).
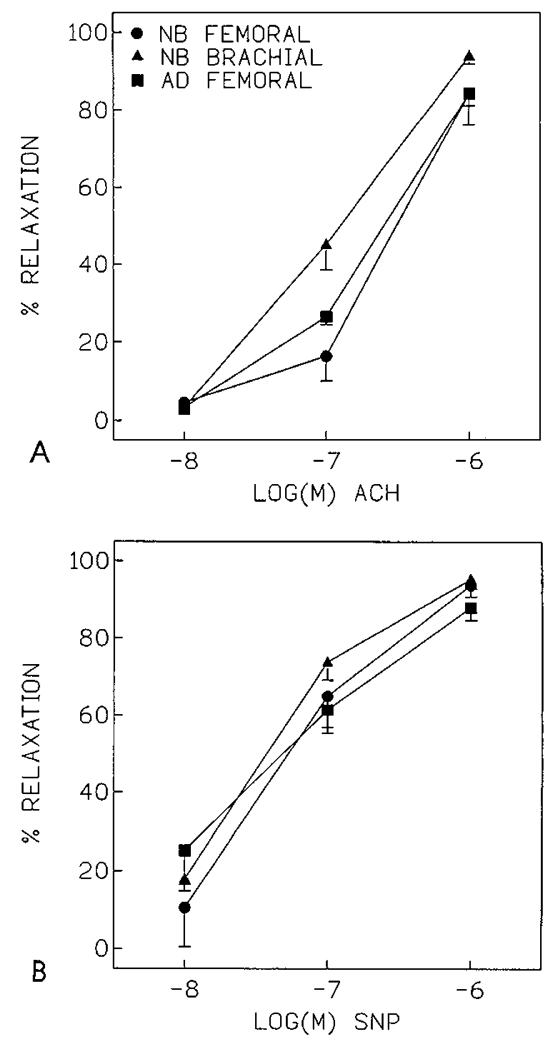

Figure 6. (A) Endothelial dependent response as percent relaxation produced by acetylcholine in vessels preconstricted with $30 \mu \mathrm{M}$ U46619 in newborn $(N B)$ femoral $(\mathbf{O})$ and brachial $(\mathbf{A})$ arteries in comparison to adult $(A D)$ femoral arteries (ם) cannulated under constant intraluminal pressure. $(B)$ Endothelial independent responses as percent relaxation of vessels preconstricted with 30 $\mu \mathrm{M} U 46619$ in response to sodium nitroprusside. Results are mean $\pm \operatorname{SEM}(n$ $=4$ per vessel group).

size, and the defects were strictly limited to hindlimbs. Although differences exist, it remains possible that hypertension is a common denominator in the pathogenesis of reduction defects after moderate hypoxia and L-NAME. It remains quite 


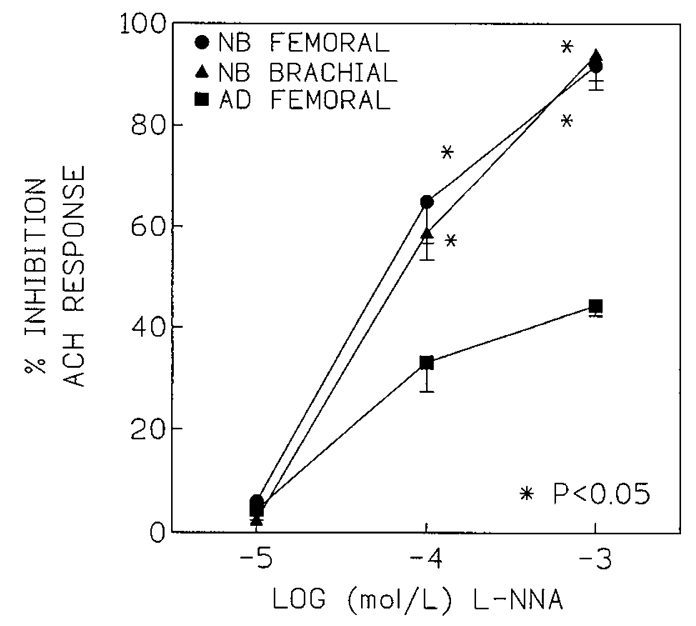

Figure 7. L-NNA-mediated inhibition of peak relaxation responses to acetylcholine. Results are expressed as percent inhibition of peak acetylcholine response in newborn $(N B)$ femoral $(\bullet)$, brachial $(\boldsymbol{\Lambda})$, and adult $(A D)$ femoral (घ) arteries. The asterisk $\left(^{*}\right)$ indicates significant $(p<0.05)$ difference in both newborn femoral and brachial arteries compared with adult femoral arteries. Results are mean $\pm \operatorname{SEM}(n=4$ per vessel group).

possible that $\mathrm{L}-\mathrm{NAME}$-mediated inhibition of NO release in the fetus produces systemic hypertension and that this is the primary factor in the genesis of LRD. In addition a regional, anatomic vulnerability to rupture and hemorrhage in the hindlimb after a systemic hypertensive response cannot be excluded in the present study. Hypertension has been shown in several clinical and experimental models to lead to endothelial impairment (18). Whether endothelial impairment is uniform or certain regions of the systemic circulation are more vulnerable to this effect is unknown. It is also conceivable that the teratogenic effects of L-NAME could primarily result from changes in placental blood flow. In addition to LRD, L-NAME did produce significant intrauterine growth retardation and decrease in placental weight (3). In contrast to the LRD, however, the intrauterine growth retardation and the reduced placental size were not prevented by concurrent administration of L-arginine or SNP which indicates a process not specific for inhibition of NO release.

Whereas myogenic tone is generally greatest in small resistance vessels, large conductance vessels as used in this study generally demonstrate little resting tone. The findings of this study suggest increased NO release may be generalized throughout the systemic circulation with particular involvement of resistance vessels or arterioles that are primarily involved in the regulation of regional blood flow. In addition, the high basal NO release was demonstrated in the absence of intraluminal flow, suggesting that in this experimental setting shear stress is not a necessary determinant of basal NO release.

LRD represent a diverse group of birth defects that are multifactorial in etiology. The thalidomide epidemic in the 1960s focused widespread public attention on the adverse effects of environmental agents (teratogens) on fetal develop- ment (19). A considerable body of knowledge has subsequently accumulated on the etiology of LRD, most of which occur as part of a chromosomal anomaly or, alternatively, the result of an environmental influence that compromises fetal circulation. Early amnion rupture and other compression syndromes account for many LRD and are attributed to mechanical interference with fetal circulation (20). Only a few teratogens whose primary effects are on vascular tone such as caffeine and cocaine have been implicated in the etiology of LRD (21). Although previous reports have speculated that certain teratogens may target the endothelium (22), this is the first study to demonstrate inhibition of endothelial NO release in the pathogenesis of LRD and clearly reflects the importance of constitutive NOS expression during normal fetal development.

Acknowledgment. The authors thank Xiao-Jing Zang, M.D., for technical assistance.

\section{REFERENCES}

1. Snyder SH, Bredt DS 1991 Nitric oxide as a neuronal messenger. Trends Pharmacol Sci 12:125-130

2. Busse R, Mulsch A, Fleming I, Hecker M 1993 Mechanisms of nitric oxide release from the vascular endothelium. Circulation 87(suppl V)18:25

3. Diket AL, Pierce MR, Munshi UK, Voelker CA, Eloby-Childress S, Greenberg SS, Zhang X-J, Clark DA, Miller MJS 1995 Nitric oxide inhibition causes intrauterine growth retardation and hindlimb disruption in rats. J Obstet Gynecol (in press)

4. Beckman DA, Brent RL 1984 Mechanisms of teratogenesis. Annu Rev Pharmacol Toxicol 24:483-500

5. Beckman DA, Brent RL 1986 Mechanism of known environmental teratogens: drugs and chemicals. Clin Perinatol 13:649-687

6. Conrad KP, Vernier KA 1989 Plasma level, urinary excretion and metabolic production of cGMP during gestation in rats. Am J Physiol 257:R847-R853

7. Conrad KP, Joffe GM, Kruszyna H, Kruszyna R, Rochelle LG, Smith RP, Chavez JE Mosher MD 1993 Identification of increased nitric oxide biosynthesis during pregnancy in rats. FASEB $\mathrm{J}$ 7:566-571

8. Baylis C 1984 Renal hemodynamics and volume control during pregnancy in the rat. Semin Nephrol 4:208-220

9. Miller MJS, Sadowska-Krowicka H, Chotinaruemol S, Kakkis JL, Clark DA 1993 Amelioration of chronic ileitis by nitric oxide synthase inhibition. J Pharmacol Exp Ther 11:16

10. Gardiner SM, Comptom AM, Bennett T, Palmer RMJ, Moncada S 1990 Regional hemodynamic changes during oral ingestion of $N^{\mathrm{G}}$-monomethyl-L-arginine or $N^{\mathrm{G}}$ nitro-L-arginine methyl ester in conscious Brattelboro rats. Br J Pharmacol 101:10-12

11. Baylis C, Mitruka B, Deng A 1992 Chronic blockade of nitric oxide synthesis in the rat produces hypertension and glomerular damage. J Clin Invest 90:278-281

12. Steel RGD, Torrie JH 1960 Principles and Procedures of Statistics. McGraw-Hill, New York, pp 51-73

13. Koller A, Sun D, Messina EJ, Kaley G 1993 L-Arginine analogues blunt prostaglandin-related dilation of arterioles. Am J Physiol 264:H1194-H1198

14. Vanhoutte PM, 1993 Other endothelum-derived vasoactive factors. Circulation 87(suppl V) $9: 17$

15. Davisson RL, Johnson AK, Lewis SJ 1994 Nitrosyl factors mediate active neurogenic hindquarter vasodilation in the conscious rat. Hypertension 23:962-966

16. Wilson JG, Fraser CF 1977 Handbook of Teratology, Vol 2. Plenum Press, New York, pp 160-162

17. Leist KH, Grauwiler J 1974 Fetal pathology in rats following uterine-vessel clamping on day 14 of gestation. Teratology 10:55-67

18. Miller MJS, Pinto A, Mullane KM 1987 Impaired endothelium-dependent relaxations in rabbits subjected to aortic coarctation hypertension. Hypertension 10:164-170

19. Lenz W 1988 A short history of thalidomide embryopathy. Teratology 38:203-215

20. Wolpert L 1976 Mechanisms of limb development and malformations. Br Med Bull 32:65-70

21. Hoyme HE, Jones KL, Dixon SD, Jewett T, Hanson JW, Robinson LK, Msall ME, Allanson JE 1990 Prenatal cocaine exposure and fetal vascular disruptions. Pediatrics 85:743-747

22. Stephens TD 1988 Proposed mechanism of action in thalidomide embryopathy. Teratology 38:229-239 